\title{
PLASTIC TREATMENT OF THE MUSCLES AND BONE IN AMPUTATION SURGERY
}

\author{
A Method Designed to Produce Physiological Conditions in the Stump
}

Rolf Dederich, Bonn, Western Germany

Director, the Orthopaedic Department, St Petrus Hospital, Bonn

Amputation surgery does not always receive the attention that it merits. We readily undertake major operations on the large cavities of the human body, and operations for reconstruction of the joints and hands, yet we are still in favour of fast and simple methods of amputation. The stumps resulting from such operations are expected to control modern and often complicated artificial limbs. Methods of plastic operation tried out in earlier years often failed because of infection. When the flap amputation technique was introduced it seemed as if the problem of amputation surgery would be solved for many generations of surgeons. Even in modern German textbooks one finds that physiological considerations are ignored, and that simple methods of surgery are preferred to the most appropriate management of the various elements of stumps. Skin suture only is recommended. It is considered that the stump may be shaped by bandaging, and that as little muscle as possible should be left. There are many who, in the belief that this will provide the solution of all limb-fitting problems, advocate cutting away the muscles drastically, especially in the belowknee stump, until no more than a skin-covered bone is left, in order that the shape of the stump will not change.

Many patients with amputations suffer from severe pain which not only hampers their walking ability but also disrupts their professional and family lives through recurring periods of inability to work. Relief may be attempted by altering the artificial limbs in preference to further surgical intervention. Yet there are many stump defects that cannot be corrected or compensated for by the artificial limb. Among them are the changes which result from the functional neglect of the muscles concerned, the ischaemic syndrome of the end of the stump, and the associated defects such as excessive mobility of the soft parts.

We can distinguish five groups of amputations, each with its own and slightly different problems: 1) war amputations; 2) amputations for traumatic lesions; 3) amputations in old people (necessitated by diabetes or vascular disease); 4) amputations for other disease processes such as neoplasms; 5) amputations occurring congenitally or performed for congenital anomalies.

In various countries the relative numbers in each group vary widely. In Germany 146,000 war amputations are in the vast majority; their number is much smaller in the English-speaking countries-especially in the United States-where amputations in old people dominate. In all countries an increase in the number of amputations resulting from trauma must be expected, and, because of the longer expectation of life, there will also be an increase in amputations in old people. German surgeons think that many stumps resulting from war amputations, which were generally performed on young soldiers, deteriorate about twenty years after the amputation, so that operative correction can no longer be avoided.

In principle the operative problem is the same for the five groups of amputees. Preparation, anaesthesia and the technique of the operation should be adapted to the needs of each individual case. However, when the surgeon's physiological views are confirmed by the results in several hundred cases, he will always strive to follow these basic principles for fashioning stumps in order to produce physiological conditions in the stump. 
Pain after amputation-Few patients who have had amputations do not experience the pain which, in Germany, is commonly called " nerve pain." The pain is recorded as occurring either in the stump itself or in the missing limb, or alternating between each, but rarely in both simultaneously. It cannot be measured or located, and it appears to affect different subjects with different intensity, so that many surgeons are inclined to view it with scepticism. The pain is, however, the very centre of the thoughts, the professional and domestic lives of many patients who have had amputations. They crave for pain-free stumps, pain-free sleep and the ability to do their work free of pain. Many patients seek relief with alcohol or with drugs. They consult a succession of doctors whose treatment proves disappointing. Patients have been known to undergo as many as thirty-five operations for the treatment of pain alone. The types of treatment available range from administration of drugs to various methods of surgical intervention. Opinions differ on the results of excision of neuromata, of division of nerves in the stump itself, or within the theca, and of interruption of autonomic pathways. Whereas Leriche did indeed believe in a peripheral cause of stump pain, many other surgeons attributed the pain, especially phantom pain, to a central fixation. Many failures of secondary amputations performed by simple surgical methods to relieve pain seem to deny the peripheral origin of the pain. During the second world war Thomsen (1942) and Watermann (1949) reported on the practice of muscle suture (Muskelschlingen) but the fundamental physiological rationale of osteoplasty and myoplasty in the construction of primary amputation stumps and in corrective operations was not established before Ertl (1949) and Mondry (1952) published their papers.

Functional changes after amputation-The soft parts and the muscles of an extremity are severed by amputation. The muscles retract like rubber bands. The farther the section from the bony origin the greater will be the retraction. The peripheral muscles roll up and cicatrise; in many cases their peripheral parts become adherent to the skin and pull it in, causing puckering and wrinkling. The division of muscles disturbs their tension and the balance of the antagonists. In amputations through the thigh the femur becomes abducted because the adductor action has been lost. Hence scars of guillotine and flap amputations which are usually performed transversely show lateral deviation (Fig. 1). The pulley action of the ilio-tibial tract is lost and weight is no longer carried axially on the bone. When pressure is no longer exerted on the neck of the femur there is rarefaction proportional to the decreased load both in the femur and in the vicinity of the hip joint, and the joint space narrows through loss of cartilage (Pauwels 1958).

The muscle of the stump loses its complementary organ, the joint distal to it, and with this its functional activity. Scarring and the lack of functional muscle activity decrease the flow of arterial blood, especially in the terminal area. At the same time the defective muscle pump cannot efficiently maintain venous return; so stasis results. The end of the stump shows all the clinical and histological evidence of a disturbance of blood flow (Fig. 1). All the stages can be observed on the skin of these areas: small brown petechiae; large areas of black and blue discoloration; para-keratoses and hyper-keratoses, and finally ulceration. The skin is often as much as 7 degrees Centigrade colder than that of the healthy limb. Treatment of the resulting pain by operations on the nerves does not attack the cause. The myoplasty procedure produces active muscles, restores their balance and some of their function, and brings the arterial and venous circulation back to normal. The stumps become muscular and warm, the haemosiderin is taken away, and the skin recovers its normal colour and growth of hair (Figs. 2 to 4 ).

The same observations apply to the division of muscles in amputation below the knee. In this case, however, we cut through two bones which are firmly connected with one another in the normal leg by proximal and distal syndesmoses. After amputation the bone stumps are abnormally mobile. It is possible to press their ends together and they will spring apart again. The head of the fibula moves in the opposite direction, being pulled outwards when the ends

VOL. 45 B, NO. I, FEBRUARY 1963 

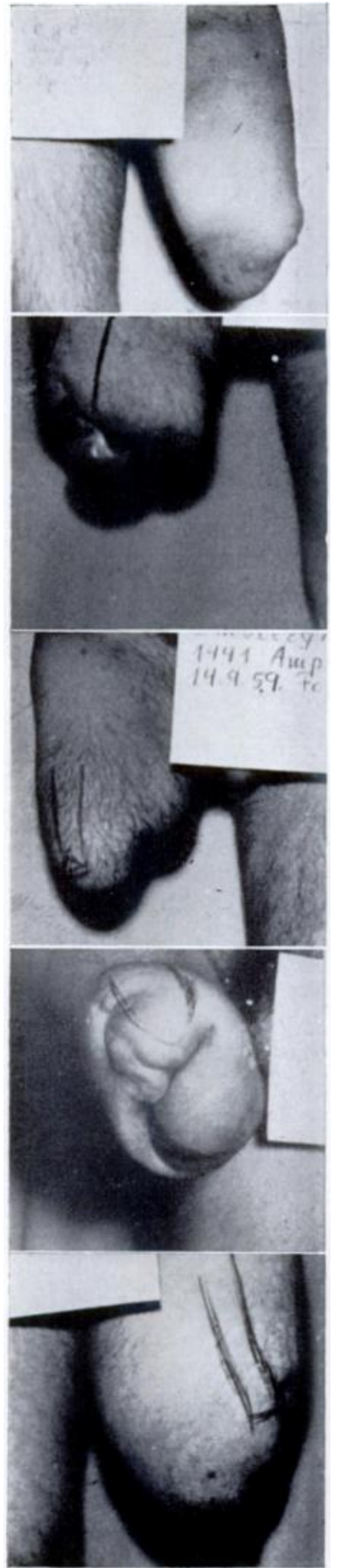

FIG. 1

Badly shaped thigh stumps. Note the protrusion of the functionless adductor muscles, the abduction of the femoral stumps and the poor circulatory condition of the terminal skin. of the bone stumps are pressed together, while the action of the muscles inserted into it tends to separate the bone ends. These phenomena happen at every step the patient takes and lead to difficulties with the artificial limb and to pressure areas, especially at the head of the fibula. These physiological considerations led Bier $(1895,1923)$ and, later, Ertl to stabilise the bone ends of the stumps of the lower leg. Mondry and I have carried out this stabilisation in several hundreds of cases with a bridge made from three roll flaps of periosteal cortical lamellae from all sides of the tibia.

\section{TECHNIQUE OF OPERATION}

A depot-antibiotic is administered on the day before the operation. For analgesia Pantocain introduced intrathecally at the space between the second and third lumbar vertebrae is preferred. If at all possible, the operation should be done with the circulation of the limb occluded by a pneumatic tourniquet. The tourniquet should not be applied in cases of vascular disease or when the stump is going to be very short. The tourniquet should be loosened before the muscle is sutured in order to ensure the best possible haemostasis.

Treatment of the bone-I emphatically reject Bunge's (1899) method. He removes a circular periosteal cylinder at the end of the severed bone and takes the bone marrow out to the same height. This procedure leads to necrosis of the bone end. Tests have shown that there is a slight positive pressure in the bone marrow which maintains venous circulation. This appears to confirm the need for sealing the marrow cavity. There are no contractile elements in the marrow cavity which could assist the venous return of the blood from its abundance in the marrow or the bones themselves. According to Loon (1960), stasis of the blood flow in the region of the bone
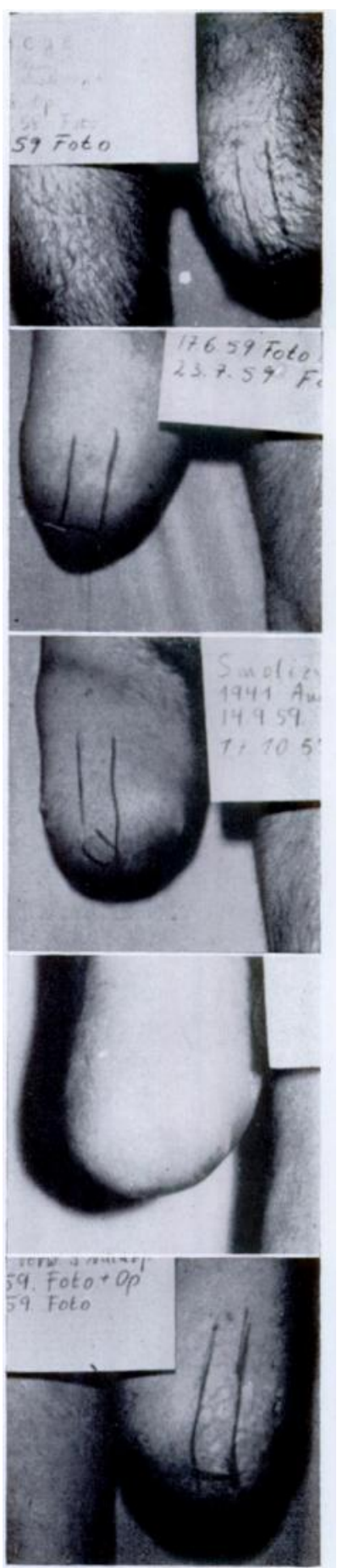

Fig. 2

The same stumps after musculoplastic stump correction. Note the restoration of the femoral stumps to the central position and the considerable improvement in the circulatory state of the terminal skin. 
end will occur when the marrow cavity is not perfectly sealed; this in turn may cause a disturbance of hydrogen ion concentration and consequent pain. For this reason we try to close the marrow cavity as tightly as possible, using periosteum and, if possible, some bone chips too. We try to sew the periosteum right around the bone end without touching the marrow and without removing it. In below-knee stumps we make the bridge between the tibia and the fibula with three periosteal flaps. In below-knee amputations the fibula should be divided rather more proximally than the tibia.

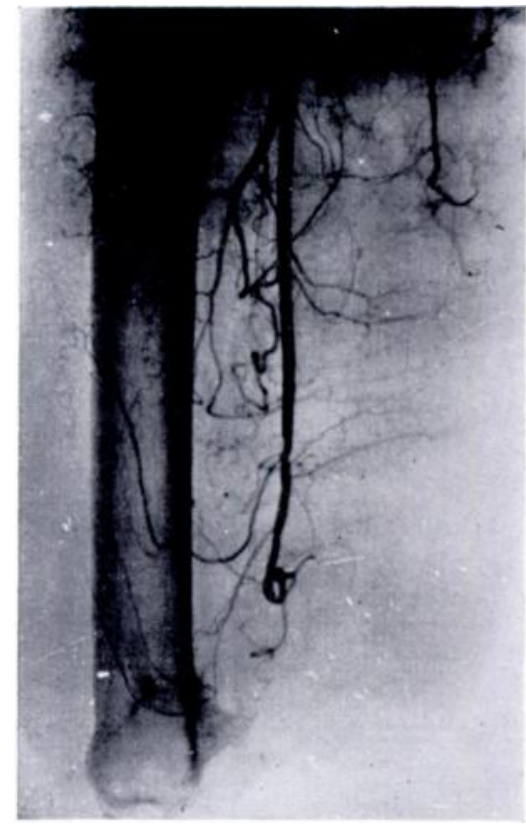

FiG. 3

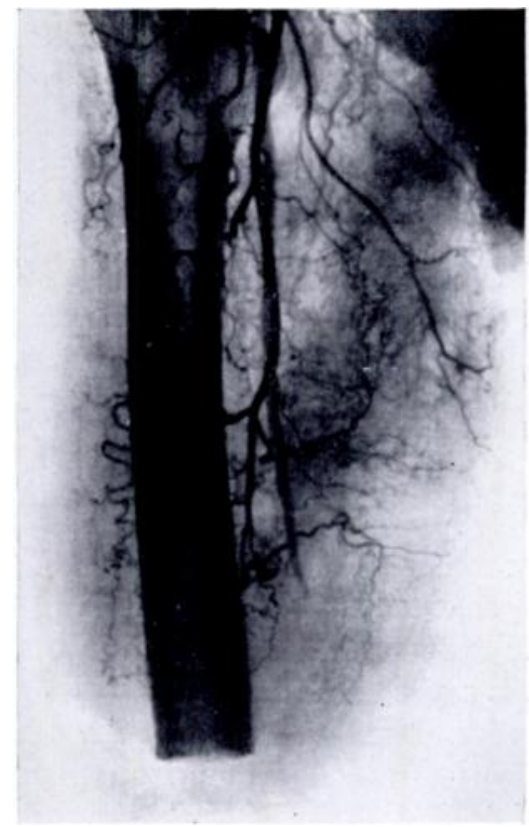

FIG. 4

Increase of arterial flow into stump after musculoplastic correction. Figure 3Arteriograph of stump showing poor filling in the adductor region and in the apex of the stump. There is no detectable filling distal to the end of the bone. Figure 4Arteriograph of the same stump ninety-five days after operation. There is greatly increased filling in all areas, especially in the adductor region and in the apex of the stump.

Treatment of the muscles-In primary amputations through the femur the muscles are separated in the shape of an obliquely positioned cross, in four functional rather than anatomical groups. In secondary operations the often considerable terminal scars have to be resected. One frequently has to look for and follow certain muscles to a depth of some inches; this applies especially to the adductors and the sartorius. One will then be able to pull them out some four to five inches (ten to twelve centimetres). The adductors must be stitched to the abductors and the ilio-tibial band over the stump of the femur. When shaping this, one must insert some sutures in front of and behind the bone to prevent the suture line from slipping off the end of the bone. The end of the bone must be completely sheathed in the muscles. This ensures that the femur does not become abducted, and that it assumes a central position within the stump. Next, the flexors and extensors are sewn together edge to edge across this obliquely positioned muscular suture line. Redundancy or overlapping of the opposing muscles should be avoided. Depending on the level of the amputation, the sartorius may be included in the group of extensors. It is sometimes possible to place these sutures in several layers. The tension of the catgut sutures must be carefully regulated because muscles with longitudinal fibres are sewn together end to end. If the tension is too great the sutures will tear. Some surgeons advise the inclusion of scar tissue or bits of fascia in the sutures in order to be able

VOL. 45 B, No. 1, februARY 1963 
to make firmer knots. However, I think it is preferable to sew together muscle which is as far as possible free from fibrous tissue.

In the below-knee stump we first sew the fibular group to the medial half of the calf muscles over the anterior portion of the bone bridge and the tibia. Sutures are placed at the front and back of the tibia so as to avoid leaving even the smallest part of the bone bridge uncovered by muscle. This suture, frequently performed in several layers, will cover about half the bone bridge with muscle. The remaining posterior half of the calf muscle is cut into a wedge and is sewn into the remaining muscle space under such tension as is permissible. In some cases it may be necessary to cut out larger wedges to prevent undesirable thickness and build-up of the end of the stump. This also applies in the case of arm stumps. In forearm stumps the muscles should be sutured as well as possible over the two bone stumps, which should be of equal length.

Treatment of the fascia-The fascia should be removed from the end of the stump in both primary and secondary operations because it is a barrier between the vessel-bearing muscles and the skin. It often shows scars as thick as a finger, forming a considerable barrier to the circulation. However, in the thigh stump the ilio-tibial band of the fascia lata should be preserved because it safeguards the pulley action on the hip joint.

Treatment of the skin and subcutaneous tissues-When the muscle suture is completed the skin is pulled out with towel clips and cut to shape in equal anterior and posterior flaps to obviate the risk of circulatory disturbances such as might arise with flaps of excessive length. It should be sewn firmly although not under tension. It is almost always possible to provide a straight transverse scar across the end of the stump, on muscle with good circulation. When the skin flaps have been cut to shape they are once more everted for the removal of all bits of scar and fascia from the dermis. The skin should not however be made too thin. In the course of this treatment the saphenous vein often becomes visible and should be tied. A short incision is made at the lowest point in the centre of the posterior flap for the suction drain. Now the various subcutaneous sutures are inserted. These should preferably incorporate any small bleeding points in the dermis. The skin is then closed with interrupted sutures. I dress the wound lightly with balsam of Peru and over this I put an elastic compression dressing with sterile paper bandages. Over this I apply a " tube-gauze " dressing. I disconnect the suction after twenty-four hours and the drain is removed after forty-eight hours. After a week I leave the wound exposed, the stump being protected by a cage. The wound will hurt for three to four days. After this time patients often complain of a sensation of tightness in the stump; this is caused by the muscle tension to which the patient is not accustomed, and may be regarded as a kind of muscular fatigue, such as is felt after excessive exercise. In this stage patients often like light traction of two to four pounds (one or two kilograms) on the skin over a tube-gauze dressing.

Patients with lower limb amputations should be kept in bed for at least three weeks to allow the sutured muscles to heal. If the patient is allowed out of bed too soon the muscle suture is endangered because the strength of the catgut decreases after a week and the mutual attachment of the muscle tissue is not yet strong enough. I do not remove the stitches until the end of the second week.

\section{AFTER CARE}

Analgesics should be given in appropriate doses during the first days after operation. Patients rarely need sedatives for longer than a week, though those who were addicts before the operation frequently show deprivation crises. These disappear after a few days. The patient has got rid of his pain and feels like a new man. Occasionally a subcutaneous accumulation of serum or blood will show up. In such cases the patients will again complain of " nerve pains." The haematoma can be aspirated, or the fluid can be let off by opening the draining channel or by releasing a stitch, and the "nerve pains" will cease. This seems 
to me further impressive proof of the fact that stump pains have peripheral rather than central causes.

\section{RESULTS}

So far the methods described have proved successful in 560 amputations and stump corrections carried out in this hospital. Stump complaints, even those of phantom pain, were relieved in almost every instance. The stumps gained muscle power and became warm. The skin recovered normal sensibility and there were no circulatory disturbances (Fig. 2). The stumps healed and the patients were free of pain and able to sleep. The amputation stump ceased to be the centre of the patient's daily life. After total contact prostheses had been fitted, the axial loading on the bone in thigh stumps caused regression of the osteoporosis and restored cartilage to the hip joint. Finally, there were failures which gave further evidence in support of amputation with plastic treatment of bone and muscle. There were a few cases in which the muscle sutures opened as a result of delayed healing. In these, unphysiological conditions developed in the end of the stumps and the patients again developed symptoms. These disappeared after the muscle connections were re-established at a second operation. In a few instances the muscle slings slipped off the tip of the bone so that the adductor and abductor suture came to lie in a position posterior to the bone. This mishap can be avoided through accurate sheathing of the tip of the bone stump during the placement of the muscle sutures.

\section{PROSTHESES}

The best prostheses for stumps so fashioned are total contact limbs and active artificial arms (McKenzie 1960). The overall adhesion of these fittings even allows the transmission of the power of isometric muscle contraction to the socket of the artificial limb. The suction space is unphysiological and can only be regarded as a "pneumothorax" in the prosthetic system. The dimensions of the space below the stump in suction sockets has been arrived at purely empirically and there is no precise knowledge about its function. A more functional attachment of the prosthesis to the stump is achieved by filling this space with a resilient foam rubber cushion or by making a total contact socket in wood, metal or plastic. The well known fact that the skin tolerates suction less well than pressure is another point against negative pressure in the suction space. In every amputation there is some danger to the venous return from the end of the stump (Hepp 1959). The artificial and unnecessary negative pressure of a suction limb adds to this danger and tends to cause the ischaemic syndrome of the end of the stump which it is our aim to avoid.

The patients do loading exercises for their stumps and also resistance exercises and phantom exercises. Strong movements of the muscles in all directions can be observed quite commonly in the end of the stump. When the stump is held firmly in a total contact socket isometric contraction of the muscles permits the patient to have a hold on the inside of the artificial limb while the amount of end loading of a contact limb gives patients a hitherto unknown and pleasant feeling of being actually in touch with the ground.

\section{SUMMARY}

1. Musculoplastic stump correction is a method of physiological preparation of all the elements of the stump-muscles, bone, vessels and nerves-so as to establish the best relations between them. It removes the cause of existing circulatory disorders by creating active muscular movements.

2. In all cases stump pains, even those of causalgic character, have been eliminated. We attribute this to the attainment of normal muscular tension, and we believe that many phantom sensations and pains are caused by cramped and retracted muscles.

VOL. 45 B, NO. 1, FEBRUARY 1963 
3. Active muscular work produces increased arterial inflow, as has been shown by arteriography, and also eliminates venous stasis in the end of the stump by restoration of the muscle pump mechanism.

4. Stumps prepared in the way described become painless and warm and their muscles become strong.

I should like to thank Dr D. S. McKenzie for his advice and encouragement and for help in translating this paper into English.

\section{REFERENCES}

BIER, A. (1895): Weitere Mittheilungen über tragfähige Amputationsstümpfe in Bereiche der Diaphysen. Archiv für Klinische Chirurgie, 50, 356.

Bier, A. (1923): Ưber Knochenregeneration, über Pseudarthrosen und über Knochentransplantate. Langenbeck Archiv für Klinische Chirurgie, 127, 1.

Bunge, R. (1899): Zur Technik und Casuistik der osteoplastischen Unterschenkelamputation nach Bier. Deutsche Medizinische Wochenschrift, 25, 353.

ERTL, J. (1949): Uber Amputationsstümpfe. Chirurg, 20, 218.

HEPP, O. (1959): Biologie des Amputationsstumpfes. Verhandlungen der Deutschen Orthopädischen Gesellschaft, 47, 391.

LeRiche, R. (1959): Die Chirurgie des Schmerzes. Leipzig: Johann Ambrosius Barth.

Loon, H. E. (1960): Prosthetics International. Copenhagen: Committee on Prostheses, Braces and Technical Aids of the International Society for the Welfare of Cripples, Pp. 48-51.

McKenzie, D. S. (1960): Personal communication.

MONDRY, F. (1952): Der muskelkräftige Ober-und Unterschenkelstumpf. Chirurg, 23, 517.

Pauwels, F. (1958): Neue Richtlinien für die chirurgische Behandlung der Coxarthrose. Langenbeck Archiv für Klinische Chirurgie, 289, 378.

Thomsen, W. (1942): Verhandlungen der Deutschen Orthopädischen Gesellschaft, 35, (unpublished).

Watermann, H. (1949): Amputationsprobleme. Zeitschrift für Orthopädie, 79, 93. 